\title{
Comparative Impact of Hearing and Vision Impairment On Subsequent Functioning
}

\author{
Margaret I. Wallhagen, PhD, RN, CS, * William J. Strawbridge, PhD, † Sarah J. Shema, MS, \\ John Kurata, PhD, and George A. Kaplan, $\mathrm{PhD} /$
}

OBJECTIVES: The purpose of this study was to compare independent impacts of two levels of self-reported hearing and vision impairment on subsequent disability, physical functioning, mental health, and social functioning.

DESIGN: A 1-year prospective cohort study.

SETTING: San Francisco Bay Area, California.

PARTICIPANTS: Two thousand four hundred forty-two community-dwelling men and women age 50 to 102 from the Alameda County Study (California).

MEASUREMENTS: Hearing and vision impairment were assessed in 1994. Outcomes, measured in 1995, included physical disability (activities of daily living, instrumental activities of daily living, physical performance, mobility, and lack of participation in activities), mental health (selfassessed, major depressive episode), and social functioning (feeling left out, feeling lonely, hard to feel close to others, inability to pay attention). All 1995 outcomes were adjusted for baseline 1994 values.

RESULTS: Both impairments had strong independent impacts on subsequent functioning. Vision impairment exerted a more wide-ranging impact on functional status, ranging from physical disability to social functioning. However, the results also highlighted the importance of hearing impairment, even when mild.

CONCLUSIONS: These impairments can be partially ameliorated through prevention, assessment, and treatment strategies. Greater attention to sensory impairments by cli-

From the "School of Nursing, University of California, San Francisco, California; ${ }^{+H}$ Human Population Laboratory, Public Health Institute, Berkeley, California; ${ }^{\ddagger}$ Northern California Cancer Center, Fremont, California; SCalifornia Department of Health Services, Berkeley, California; and "School of Public Health, University of Michigan, Ann Arbor, Michigan.

This research was supported by grant 1R37AG11375 from the National Institute on Aging and by the Prevention Health Services Block Grant from the Centers for Disease Control and Prevention.

This research was presented, in part, at the Annual Meeting of the Gerontological Society of America, November 1999, San Francisco, California. Address correspondence to Margaret I. Wallhagen, PhD, RN, CS, GNP, Department of Physiological Nursing, School of Nursing, University of California, San Francisco, CA 94143. nicians, patients, public health advocates, and researchers is needed to enhance functioning in older adults. $\mathbf{J}$ Am Geriatr Soc 49:1086-1092, 2001.

Key words: hearing loss; vision loss; disability; cohort study

$\mathrm{H}$ earing and vision impairments can disrupt interpersonal relations and limit participation in desired roles, both of which are critical to the well-being of older persons. Unfortunately, these impairments often receive minimal attention; in addition, Medicare does not routinely cover the costs of assistive devices such as eyeglasses or hearing aids. ${ }^{1,2}$

Hearing loss ranks as the third-most-prevalent major chronic disability in persons age 65 and older, being experienced by approximately $24 \%$ of those age 65 to $74,33 \%$ of those 70 and older, and up to $39 \%$ of those over the age of $75 . .^{3,4}$ It has also shown a near doubling in ageadjusted prevalence over the past 30 years in the United States and is higher at all adult ages for males than for females. ${ }^{4-8}$ Although these statistics are from studies that used self-report to assess impairment, the high prevalence of hearing impairment among older persons, its sharp increase with age, and its higher prevalence among men than women have been supported by a community-based study that used pure-tone audiometric testing, with hearing loss defined as an average loss over four frequencies $>25$ decibels in the worse ear. ${ }^{9}$

Hearing impairment is also becoming increasingly prevalent at younger ages, ${ }^{8}$ a finding that may have significant subsequent implications for health and functioning in old age. Hearing impairment alters one's ability to communicate with others and is associated with multiple negative outcomes, including physical disability, depression, loneliness, altered self esteem, and diminished functional status. ${ }^{10-15}$

Vision impairment is also of growing concern. Of individuals age 70 and older, $18 \%$ report vision impairment, with its prevalence increasing from approximately $7 \%$ at 
age 71 to $39 \%$ in those age 90 and older. ${ }^{4,16}$ Although visual functioning changes with age, most changes are amenable to correction with glasses and do not inhibit daily activities to a great extent. Major causes of vision impairment that interfere with normal activities include cataracts, glaucoma, diabetic retinopathy, and macular degeneration. ${ }^{16}$ Services and care for persons with visual impairment now cost $\$ 22$ billion annually. ${ }^{16}$ This latter statistic suggests the potential impact of vision impairment on functional status, an assumption that is supported by a number of studies. In a random sample of men and women between the ages of 65 and 84, binocular visual acuity that was worse than 20/40 was found to affect all areas of functioning assessed: activities of daily living (ADLs), instrumental activities of daily living (IADLs), physical functioning, and social interaction. ${ }^{17}$ In addition, Kalina ${ }^{16}$ reported that, in one national survey, blindness was feared more than any other physical impairment.

However, data suggest that many detrimental outcomes related to both hearing and vision impairment may be mitigated by appropriate interventions. In persons with hearing impairment, behavioral approaches that enhance coping strategies and the use of assistive devices have been shown to improve quality of life and social functioning. ${ }^{18,19}$ Brenner et al..$^{20}$ documented that improved visual acuity secondary to cataract surgery was accompanied by significant changes in quality of life. Studies support the benefits of vision rehabilitation and the use of low-vision aids. ${ }^{21,22}$ Thus, a better understanding of how these impairments influence functioning and well-being may enhance our ability to develop creative approaches to minimize their impact.

Unfortunately, although both vision and hearing impairments have been shown to significantly affect functioning, few studies have directly compared their effects. As suggested by the national survey referred to above, most unimpaired persons feel vision impairment would be worse. However, hearing impairment most directly affects communication with others. Vision impairment is often more obvious than hearing impairment, so others may have more patience with someone experiencing the former than the latter. One earlier comparison study that included only one outcome measure concluded that visual impairment was associated with subsequent disability in ADLs whereas hearing impairment was not. ${ }^{14} \mathrm{~A}$ more recent study compared the relative impacts of vision and hearing impairment on several types of physical disability, reporting that vision impairment had a stronger impact on several forms of physical disability; ${ }^{23}$ however, disability had not been assessed at baseline, making it difficult to adequately assess causal order. ${ }^{24}$

This paper presents data from a longitudinal study demonstrating the significant independent relative impacts of both hearing and vision impairment on physiological and psychosocial functioning in older adults. Both forms of impairment are included in each statistical model so that the independent impacts of each can be assessed. Adjustments are included for all outcome variables at baseline, allowing for a better assessment of causal order. Data are discussed in light of other research findings; they support the need for further research to reduce the incidence of hearing and vision impairment, and the need for the uti- lization of current and development of new interventions to minimize their impact when they occur.

\section{METHODS}

\section{Study Population}

A longitudinal study of factors related to health and mortality, the Alameda County Study began in 1965 by enrolling 6,928 persons age 16 to 94 . Subjects were selected by means of a random household survey in Alameda County, California, an area that includes the cities of Oakland and Berkeley. The county had been selected in 1965 partly on the basis of having similar demographic characteristics to the United States as a whole. Data collection has been by means of a mailed questionnaire. Participants were resurveyed in 1974, 1983, 1994, and 1995. Response rates for these four follow-up surveys were $85 \%, 87 \%, 93 \%$, and $97 \%$, respectively. Over 2,000 subjects have died since the study began.

Those eligible for the analyses of hearing and vision impairment reported in this paper included the 2,504 subjects age 50 and older in 1994 who completed both the 1994 and 1995 questionnaires; 62 of these were subsequently excluded because they were missing one or more responses to the hearing or vision assessment questions or to any of the adjustment variables. The total remaining was 2,442 . A few subjects ( 2 to 27 ) with missing data on individual outcome measures were omitted from individual statistical models where the missing values occurred. Minorities constituted $17 \%$ of the subjects: African Americans $8 \%$, Hispanics $4 \%$, Asians 4\%, and Native Americans $1 \%$.

\section{Measures}

The 1994 questionnaire was designed to assess a wide variety of behavioral, medical, and social factors, whereas the shorter 1995 questionnaire emphasized physical functioning, mental health, morale, and social relationships. Outcomes analyzed in the analyses that follow are limited to those included in both questionnaires.

\section{Hearing Impairment}

Subjects were asked in 1994 how much difficulty they had (even with a hearing aid) hearing and understanding words in a normal conversation, hearing words clearly over the telephone, and hearing well enough to carry on a conversation in a noisy room. Response sets used for assessing level of hearing difficulty were "a great deal" (3), "some" (2), "a little" (1), or "none" (0). Scores were summed. The resulting scale was then divided into three categories: no hearing impairment (score of 0 ), mild hearing impairment (score of 1-3), and moderate or more hearing impairment ( 4 or higher). The rationale for this scoring was that the break for moderate or more hearing impairment should involve difficulty in more than one setting plus at least one report of a great deal of difficulty or two reports of some difficulty.

\section{Vision Impairment}

Three questions were also used to assess vision impairment. Subjects were asked how much difficulty they had (even with glasses) seeing well enough to read street signs 
at night, recognize a friend across the street, and read a newspaper. The same response sets and scoring algorithms were used to categorize vision impairment as described above for hearing impairment.

\section{Functioning Outcomes}

All 1995 outcomes had also been assessed in 1994. Variables were dichotomized for use with logistic regression analysis because all but two variables either involved only two response options on the questionnaire or were scored dichotomously according to normal usage, such as the disability variables, depression, and self-rated health. The two variables used to assess social functioning (feel lonely or remote, and can't pay attention) could potentially be used as a scale, but the modal responses for all three variables were at one end of the distribution, effectively excluding the use of multiple regression.

Physical health was measured by self-rated physical health. Those reporting it as fair or poor were compared with those reporting it as good or excellent.

Physical functioning included disability in ADLs, IADLs, and physical performance. ADL disability was defined as reporting any trouble with or needing help walking across a small room, bathing, grooming, eating, dressing, transferring from bed to chair, or using the toilet. IADL disability was defined as reporting any trouble with cooking, heavy housework, shopping, or managing money. Physical performance disability was defined as having a lot of difficulty or needing help with pulling or pushing large objects, writing, handling small objects, standing up after sitting in a chair, getting up from stooping or kneeling, reaching or extending arms above the shoulder, lifting or carrying weights over 10 pounds, stooping, crouching, or kneeling. The more severe category of a lot of difficulty was used for the physical performance items because many functional middle-aged adults report a little or some difficulty with one or more of these items. ${ }^{25}$ Mobility disability was defined as reporting any difficulty walking one quarter of a mile without help or walking up 10 steps without resting. Never participating in activities was defined as never going out to entertainment, sports events, community, or volunteer activities.

Mental health included two variables. Depression was measured using the 12 items and scoring algorithm from the Diagnostic and Statistical Manual Third Edition Revised (DSM-III-R) major depressive episodes scale. ${ }^{26}$ Those scored as depressed were compared with those not depressed. Selfrated mental health was measured with a question asking subjects to evaluate their own mental health and was scored in the same way as self-rated physical health.

Social functioning included four variables. Hard to feel close to others and feel left out even in a group involved simple true/false responses. For each, those saying the statement was true were compared with those saying it was false. The third item (feel lonely or remote) compared those answering sometimes or often with those answering never. Can't pay attention compared those saying they had difficulty paying attention sometimes or often with those saying never.

\section{Baseline Chronic Conditions and Other Adjustment Variables}

Chronic conditions from the 1994 survey included presence in the last 12 months of heart disease, high blood pressure, stroke, transient ischemic attack, diabetes mellitus, cancer, peripheral vascular disorders, bronchitis, and emphysema. Scoring was 0,1 , or 2 or more conditions reported. Age was measured in years. Education was coded as 12 years or more versus less, marital status as married versus all other responses, and ethnicity as African American versus all other categories.

\section{Statistical Analysis}

Separate sensory impairment prevalence rates were calculated by 10-year age period for each gender based upon the divisions of the two scales into the three categories of no impairment, mild impairment, and moderate or more.

Using logistic regression, each 1995 outcome was then regressed on age, gender, ethnicity, education, marital status, chronic conditions, and the two sets of impairment variables. For both hearing and vision impairment, the two levels of 1994 impairments were coded as indicator variables; the reference categories were no hearing or vision impairment, respectively. Dose-response patterns were tested in separate models using indicator variables coded to represent comparisons between adjacent impairment levels (mild compared with none, moderate compared with mild). ${ }^{27} \mathrm{~A}$ dose-response pattern was considered to have occurred if both coefficients for the comparison variables were positive and statistically significant. ${ }^{28}$

In each statistical model, the 1994 measure for the same 1995 outcome under study was included to remove any cross-sectional association at baseline between each type of impairment and the outcome under investigation. In addition, by entering both impairments in the same model, the analysis allowed for the assessment of the independent or unique impact of each impairment over and above any associated with the other. Ninety-five percent confidence intervals were used with all odds ratios.

All statistical analyses were performed with the use of SAS 6.12 software.

\section{RESULTS}

Tables 1 and 2 present the prevalence of hearing and vision impairments by age and gender for the 2,442 respondents. Prevalence of both forms of impairment rises sharply with age. A slightly higher proportion of respondents reported at least some vision impairment $(51.6 \%)$ than hearing impairment $(48.5 \%)$, but more individuals reported moderate or more hearing impairment $(17.1 \%)$ than moderate or more vision impairment $(12.3 \%)$. Gender comparisons indicate a higher prevalence of hearing impairment (mild and moderate or more) for males $(57.3 \%$ vs $41.7 \%$ ) but a higher prevalence of vision impairment (mild and moderate or more) for females $(57.1 \%$ vs $44.5 \%$ ). Of those with moderate or more hearing impairment, $31 \%$ reported moderate or more vision impairment, whereas $43 \%$ of those with moderate or more vision impairment also reported moderate or more hearing impairment.

Results of the 1-year outcome comparisons are shown in Table 3. Both forms of impairment are associated with a wide range of functional outcomes. The odds ratios for the physical disability measures are higher for moderate or more vision impairment than for hearing impairment on four of the five measures, although mild hearing impairment has a greater impact on ADL performance than mild 
Table 1. Prevalence of Hearing Impairment in 1994 by Age and Gender for 2,442 Alameda County Study Subjects Age 50 to 102

\begin{tabular}{lrrr}
\hline & \multicolumn{3}{c}{ Level of Hearing Impairment } \\
\cline { 2 - 4 } Group & \multicolumn{1}{c}{ None } & Mild & Moderate or more \\
\hline By Age & & & \\
$50-59(\%)$ & $545(60.8)$ & $263(29.3)$ & $89(9.9)$ \\
$60-69(\%)$ & $390(54.8)$ & $216(30.3)$ & $106(14.9)$ \\
$70-79(\%)$ & $248(41.0)$ & $214(35.4)$ & $143(23.6)$ \\
80 and older (\%) & $76(33.3)$ & $73(32.0)$ & $79(34.6)$ \\
By Gender & & & \\
Women (\%) & $805(58.3)$ & $410(29.7)$ & $166(12.0)$ \\
Men (\%) & $454(42.8)$ & $356(33.6)$ & $251(23.7)$ \\
All (\%) & $1,259(51.6)$ & $766(31.4)$ & $417(17.1)$ \\
\hline
\end{tabular}

vision impairment. For the two mental health measures, the odds ratios are also higher for the impact of moderate or more vision impairment than hearing impairment, although even moderate or more hearing impairment still has an important effect on both outcomes. For social functioning, both forms of impairment exert significant impacts, with mild hearing impairment showing stronger effects than mild vision impairment on feeling left out, feeling lonely or remote, and can't pay attention. The results suggest a dose-response pattern. However, the statistical tests for dose response indicated that only four sets of odds ratios met the necessary criteria and these were all for vision impairment: ADL disability, never participate in activities, fair or poor mental health, and can't pay attention.

\section{DISCUSSION}

These analyses support the importance of vision and hearing impairments as growing concerns. By the age of 70 to 79 , approximately $24 \%$ of Alameda County Study participants were experiencing moderate or more hearing impairment and $16.5 \%$ were experiencing moderate or more vision impairment. These proportions increased to almost $35 \%$ and $30 \%$, respectively, in those age 80 and older. Even by the age of 50 to 59 , approximately $29 \%$ of study

Table 2. Prevalence of Vision Impairment in 1994 by Age and Gender for 2,442 Alameda County Study Subjects Age 50 to 102

\begin{tabular}{lrrr}
\hline & \multicolumn{3}{c}{ Level of Vision Impairment } \\
\cline { 2 - 4 } Group & \multicolumn{1}{c}{ None } & Mild & Moderate or more \\
\hline By Age & & & \\
$50-59(\%)$ & $488(54.4)$ & $338(37.7)$ & $71(7.9)$ \\
$60-69(\%)$ & $355(49.9)$ & $295(41.4)$ & $62(8.7)$ \\
$70-79(\%)$ & $256(42.3)$ & $249(41.2)$ & $100(16.5)$ \\
80 and older (\%) & $83(36.4)$ & $77(33.8)$ & $68(29.8)$ \\
By Gender & & & \\
Women (\%) & $593(42.9)$ & $591(42.8)$ & $197(14.3)$ \\
Men (\%) & $589(55.5)$ & $368(34.7)$ & $104(9.8)$ \\
All (\%) & $1,182(48.4)$ & $959(39.3)$ & $301(12.3)$ \\
\hline
\end{tabular}

participants were experiencing mild hearing impairment and almost $38 \%$ were experiencing mild vision impairment. Thus, any negative effect exerted on functioning could have a significant impact on healthcare utilization and quality of life.

Although comparisons of prevalence rates are difficult because of different methods of assessment and age grouping, the findings of this study are consistent with rates reported elsewhere. $3,4,9,16$ Similar to previous data, our findings support the higher prevalence of hearing impairment in men than in women. ${ }^{3,4,9}$ However, less expected was the higher prevalence of vision impairment in women than in men. Although often not reported, this finding is supported by other data. In their surveillance for sensory impairment and its impact on older adults, Campbell et al. ${ }^{4}$ noted that men were less likely than women to report vision impairments, and earlier data from the National Health Survey reporting on eye care visits and use of corrective lenses found that the level of corrective lens use was higher among females than males at all age levels above 5 years of age. ${ }^{29}$ Although the reason for this difference is not clear, some data are suggestive. Saaddine et al. ${ }^{30}$ found a higher prevalence of self-rated vision impairment among adult women with diabetes mellitus than among men with diabetes mellitus; even after adjusting for age and type of diabetes mellitus, the odds of having vision impairment were $30 \%$ higher for women than men. In addition, a study reported by Kalina ${ }^{16}$ found that visually significant cataract was higher in women than men, with a prevalence in those age 75 and older reaching $45.9 \%$ in women versus $38.8 \%$ in men. Finally, age-related macular degeneration is more common in women than men. ${ }^{16}$ These data need further validation but suggest that women may be more predisposed to a number of the major causes of vision impairment that interferes with normal activities such as cataracts, diabetic retinopathy, and macular degeneration.

How valid are self-report measures of sensory impairment? Several comparative studies support the validity of self-report data. Reuben et al. ${ }^{31}$ compared self-report with clinical testing for hearing impairment and found that a scale derived from simple questions incorporating sociodemographic data (age, gender, education) along with reported hearing difficulty (uncorrected) was an effective screen for hearing impairment. In the analyses of Rudberg et al., ${ }^{14}$ clinical and self-report measures of both forms of sensory impairment had relatively close agreement when used as predictors of subsequent disability. Although selfreport and clinical testing of sensory impairments may capture slightly different aspects of these phenomena, the fact that our prevalence data are similar to those found by others is supportive of the results.

Although further study of the relationship between clinical testing and self-report are warranted, the findings of the current study have important implications. The comparative results reported here suggest that vision impairment exerts a more wide-ranging impact on functional status, ranging from physical disability to social functioning. However, the results also highlight the importance of hearing impairment, even when mild. Thus, even if not consistently statistically significant, the odds ratios suggest a negative impact on functioning across domains for both 
Table 3. Logistic Regression Models on 1995 Outcomes for Two Levels of 1994 Hearing and Vision Impairment $(\mathrm{N}=2,442)$

\begin{tabular}{|c|c|c|c|c|c|c|c|c|}
\hline \multirow[b]{3}{*}{1995 Outcome } & \multicolumn{4}{|c|}{ Hearing Impairment } & \multicolumn{4}{|c|}{ Vision Impairment } \\
\hline & \multicolumn{2}{|c|}{ Mild } & \multicolumn{2}{|c|}{ Moderate/more } & \multicolumn{2}{|c|}{ Mild } & \multicolumn{2}{|c|}{ Moderate/more } \\
\hline & OR & $95 \% \mathrm{Cl}$ & OR & $95 \% \mathrm{Cl}$ & OR & $95 \% \mathrm{Cl}$ & OR & $95 \% \mathrm{Cl}$ \\
\hline \multicolumn{9}{|l|}{ Self-rated health } \\
\hline Fair to poor & 1.19 & $0.85-1.65$ & 1.25 & $0.85-1.84$ & 1.02 & $0.74-1.39$ & 1.63 & $1.07-2.48$ \\
\hline \multicolumn{9}{|l|}{ Physical disability } \\
\hline ADL & 1.61 & $1.14-2.28$ & 1.45 & $0.96-2.18$ & 1.50 & $1.07-2.10$ & 2.29 & $1.49-3.52$ \\
\hline IADL & 1.17 & $0.91-1.51$ & 1.22 & $0.88-1.68$ & 1.45 & $1.15-1.84$ & 1.79 & $1.25-2.58$ \\
\hline Physical performance & 1.12 & $0.81-1.54$ & 1.69 & $1.15-2.48$ & 1.25 & $0.92-1.70$ & 1.64 & $1.09-2.47$ \\
\hline Mobility & 1.21 & $0.88-1.66$ & 1.07 & $0.72-1.58$ & 1.58 & $1.16-2.14$ & 1.42 & $0.93-2.18$ \\
\hline Never participate in activities & 0.80 & $0.56-1.13$ & 0.76 & $0.50-1.16$ & 1.32 & $0.95-1.84$ & 1.91 & $1.23-2.95$ \\
\hline \multicolumn{9}{|l|}{ Mental Health } \\
\hline Fair or poor & 1.28 & $0.91-1.82$ & 1.54 & $1.03-2.30$ & 1.37 & $0.97-1.92$ & 2.45 & $1.60-3.75$ \\
\hline Depressed & 1.12 & $0.77-1.63$ & 1.64 & $1.07-2.52$ & 1.27 & $0.89-1.83$ & 2.15 & $1.38-3.35$ \\
\hline \multicolumn{9}{|l|}{ Social functioning } \\
\hline Feel left out & 1.52 & $1.14-2.03$ & 1.66 & $1.16-2.38$ & 1.47 & $1.11-1.94$ & 1.69 & $1.16-2.48$ \\
\hline Feel lonely or remote & 1.25 & $1.01-1.55$ & 1.27 & $0.95-1.68$ & 1.09 & $0.89-1.34$ & 1.51 & $1.10-2.10$ \\
\hline Hard to feel close & 1.16 & $0.89-1.51$ & 1.55 & $1.11-2.16$ & 1.16 & $0.90-1.50$ & 1.47 & $1.02-2.12$ \\
\hline Can't pay attention & 1.46 & $1.18-1.79$ & 1.73 & $1.31-2.29$ & 1.33 & $1.09-1.62$ & 1.82 & $1.33-2.50$ \\
\hline
\end{tabular}

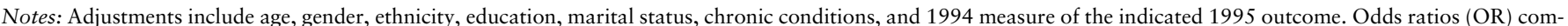
pare prevalence of each outcome for subjects at two levels of hearing and vision impairment with those reporting no respective impairment.

$\mathrm{CI}=$ confidence interval; $\mathrm{ADL}=$ activities of daily living; IADL $=$ instrumental activities of daily living.

mild and moderate or more hearing impairment. Of most importance is the impact of hearing impairment on social functioning, with persons reporting even a mild hearing impairment being more likely to feel lonely, remote, or left out and have more difficulty paying attention. Given the importance of social contacts and relationships to wellbeing in old age, these findings are cause for concern.

Both sensory impairments had strong negative impacts on mental health. For those with a moderate or more vision impairment, the odds ratios for feeling depressed and reporting fair or poor mental health were 2.15 and 2.45 , respectively. For those with moderate or more hearing impairment the odds ratios for these same two outcomes were 1.64 and 1.54. Although not reaching statistical significance, even mild hearing or vision impairment follows a similar negative pattern, with odds ratios ranging from 1.12 to 1.37 . The lack of statistical significance may relate to several factors. Depression was assessed using DSM criteria for clinical depression and the number of individuals with clinical depression was small. Thus further assessment may be important using additional measures of depressive sympotomatology and subsyndromal depression. In addition, these findings may suggest a potential dose-response effect and emphasize the importance of intervening at earlier stages of hearing and vision impairment to prevent progression.

Our findings of a negative impact on mental health are partially supported by a cross-sectional study carried out in Italy by Carabellese et al. ${ }^{32}$ They assessed 1,191 noninstitutionalized people age 70 to 75 and found both hearing and visual impairment to be associated with an increased risk of depression, although a stronger association was found with visual impairment. In contrast to our findings, visual impairment (but not hearing impairment) was associated with fewer social relationships. In a follow-up study, Appollonio et al. ${ }^{33}$ found that both uncorrected hearing and visual impairment were associated with significant impairment in mood, self-sufficiency in IADLs, and social relationships. Correction through the use of sensory aids appeared to mitigate these negative effects. Our data do not allow us to evaluate the impact of corrective sensory aids or adaptive strategies. However, because the questions elicited how much difficulty subjects were experiencing even with a hearing aid or glasses, sensory aids, if being used, are not adequately correcting experienced deficits.

In another study, Dargent-Moina et al. ${ }^{34}$ found that both visual deficits and serious hearing impairment negatively affected physical independence in women. In contrast, a more narrowly defined study accomplished by Rudberg et al. ${ }^{14}$ found that visual impairment, but not hearing impairment, was associated with ADL disability. This latter study included controls for selected chronic illnesses and baseline disability but did not assess psychosocial outcomes. In a recent surveillance summary based on population data, both vision and hearing impairments negatively affected physical functioning, although hearing impairment had a less extensive effect. ${ }^{4}$ In this surveillance, hearing impairment did not impede getting together with relatives or eating at restaurants, and those with a hearing impairment were only slightly less likely than those without a hearing impairment to get together with friends.

The conflicting findings from previous studies may be explained partially by varying research designs and inconsistencies in the measurement of hearing and vision impairment. In general, most studies have been cross-sectional, limiting the ability to identify causal sequencing. A related issue is lack of baseline adjustments for outcome 
measures, thus confounding any relationships identified. In addition, many community-based studies rely on a single question (such as asking subjects whether they have trouble hearing) or use only one level of impairment rather than attempting to assess various degrees of such loss. Findings from the current study strengthen the link between both hearing and vision impairment and reduced physical and psychosocial functioning through use of a longitudinal approach, controls for baseline assessments, use of two levels of impairment, and testing for a doseresponse effect.

An important finding in the current study is that even mild impairments exert a toll on older adults. Given the growing awareness of the importance of continued activity for the maintenance of physiological health in old age, restrictions in ADLs and mobility may exert a double effect; initially activities and contacts are limited, which can negatively affect social relationships and mental health, but eventually a cycle of increasingly poor physical status related to disuse and deconditioning may evolve. Interventions to prevent immobility may be critical. In addition, restricted activities may limit social contacts, which may contribute to isolation and poor mental health. Reinhardt's ${ }^{35}$ finding supports this idea that friendship support, independent of family support, is important for adaptation to chronic visual impairment.

The relationship between sensory impairment and functioning may also be indicative of a more centrally controlled process. Although many older adults remain cognitively intact, the prevalence of dementia, mild cognitive impairment, and memory complaints all increase sharply with age. Of potential significance is that several studies have found a significant relationship between sensory functioning, as represented by visual and auditory acuity and cognitive functioning in older adults. ${ }^{36,37}$ Luszcz and Bryan $^{38}$ suggest that cognitive functioning may depend on the integrity of sensory function as a critical neurophysiological resource, but both may represent alterations in neurological integrity. These data raise the issue of whether interventions that maintain neurological integrity would minimize sensory and cognitive changes ${ }^{39}$ and emphasize the potential importance of research that would delineate the mechanisms involved in sensory impairment and interventions to delay or prevent their occurrence. However, Gennis et al. $^{40}$ found no evidence of a major impact of hearing acuity on cognitive function in a 5-year longitudinal study of healthy older men and women. Thus, further research is needed that integrates research in these important areas.

From a healthcare perspective, these analyses support the need for further research into the mechanisms that lead to vision and hearing impairment so as to prevent their occurrence, and for the development of interventions to minimize their impact. Risk factors that have been identified include occupational noise exposure, pharmacotherapeutic agents, industrial chemicals, rapid changes in ambient pressure, and a number of medical conditions, such as diabetes mellitus, ear infections, and cardiovascular disease. , $^{7,41-47}$

Regulations have reduced occupational exposure, but more data are needed on the long-term effects of low to moderate noise exposure. Although the levels may contrib- ute more to stress than hearing loss, short bursts of high intensity noise may show cumulative effects. In addition, with the increasing use of antibiotics, antihypertensives, and other chemotherapeutic agents, greater attention needs to be given to their potential impact on subsequent hearing impairment, especially when used long term. ${ }^{48}$

The impact of hearing impairment can also be minimized through the use of current technology. Although they never fully correct the problem, hearing aids can significantly improve quality of life and social functioning ${ }^{19}$ and options have expanded. However, individuals need to understand that adaptation to hearing aids takes time, patience, and persistence and needs to be guided by a specialist. Practitioners can facilitate this process by preparing individuals in advance and providing on-going support and encouragement over the first several months.

Aside from hearing aids, other approaches to enhancing the functioning of persons who are hearing impaired are often overlooked. These include assistive devices such as amplifiers, telephone attachments, computer-assisted communication, decoders that allow for viewing closed caption television, and hearing ear dogs. ${ }^{19}$ They also include teaching older adults how to cope more effectively with their hearing impairment. For example, Andersson et al. ${ }^{18}$ demonstrated the benefits of a behavioral group treatment program that included applied relaxation, video self-modeling, exposure, information, and various coping skills.

Changes in visual acuity with age are almost universal findings. However, the influence of other age-related contributors, including cataracts, glaucoma, macular degeneration, and diabetic retinopathy, can be modified through programs aimed at prevention (including tight glycemic control in diabetics and limiting exposure to free radicals), early detection, and treatment. For example, Brenner et al. ${ }^{20}$ demonstrated the positive effect of cataract surgery and other treatments to improve visual functioning on quality of life.

Visual rehabilitation is also a critical element of care. Studies support the benefits of visual rehabilitation on functioning. ${ }^{21,22,49}$ Visual aids such as magnifiers or referral to resources such as talking books may be key; even attention to lighting in the individual's home environment can enhance quality of life and maximize function. ${ }^{50} \mathrm{Ap}$ pollonio et al. ${ }^{33}$ found that persons with corrected sensory impairments (hearing and vision) did not experience changes in mood or self-sufficiency in IADLs or social relationships and had lower mortality rates than persons with uncorrected sensory impairments. Unfortunately, many resources that might be available for hearing and visionimpaired individuals are not covered by insurance.

In summary, data from the current study provide additional evidence that hearing and vision impairments have significant negative impacts on quality of life and functioning for older persons. Of particular clinical relevance is the impact of even mild impairments that might often go unrecognized or untreated. Although further research is needed to refine our understanding of the factors that contribute to these sensory impairments, clinicians can have a positive impact by stressing prevention and early assessment amd facilitating use of assistive devices and techniques for ameliorating losses that have already occurred. 


\section{REFERENCES}

1. Ettinger WH. The health care system for older adults in the United States. In: Hazzard WR, Blass JP, Ettinger WH et al., eds. Principles of Geriatric Medicine and Gerontology. New York: McGraw-Hill, Inc, 1999, pp 457-466.

2. Reuben DB, Yoshikawa TT, Besdine RW. Geriatrics Review Syllabus: A Core Curriculum in Geriatric Medicine. New York: American Geriatrics Society, 1996.

3. Rees TS, Duckert LG, Carey JP. Auditory and vestibular dysfunction. In: Hazzard WR, Blass JP, Ettinger WH et al., eds. Principles Of Geriatric Medicine and Gerontology. New York: McGraw-Hill, Inc., 1999, pp 617-631.

4. Campbell VA, Crews JE, Moriarty DG et al. Surveillance for sensory impairment, activity limitation, and health-related quality of life among older adults-United States, 1993-1997. Morb Mortal Wkly Rep CDC Surveill Summ 1999;48:131-156.

5. Current estimates from the National Health Interview Survey, 1993. Vital Health Stat 1994;10(190):1-221.

6. Jackson AL. Prevalence of selected impairments. Vital Health Stat 11968 10(48):1-78.

7. Ries PW. Prevalence and characteristics of persons with hearing trouble: United States, 1990-91. Vital Health Stat 1994;10(188):1-75.

8. Wallhagen MI, Strawbridge WJ, Cohen RD et al. An increasing prevalence of hearing impairment and associated risk factors over three decades of the Alameda County Study [see comments]. Am J Public Health 1997;87:440-442.

9. Cruickshanks KJ, Wiley TL, Tweed TS et al. Prevalence of hearing loss in older adults in Beaver Dam, Wisconsin. The Epidemiology of Hearing Loss Study. Am J Epidemiol 1998;148:879-886.

10. Chen HL. Hearing in the elderly. Relation of hearing loss, loneliness, and self-esteem. J Gerontol Nurs 1994;20:22-28.

11. Dugan E, Kivett VR. The importance of emotional and social isolation to loneliness among very old rural adults. Gerontologist 1994;34:340-346.

12. Jerger J, Chmiel R, Wilson $\mathrm{N}$ et al. Hearing impairment in older adults: New concepts. J Am Geriatr Soc 1995;43:928-935.

13. Mulrow CD, Aguilar C, Endicott JE et al. Association between hearing impairment and the quality of life of elderly individuals. J Am Geriatr Soc 1990; 38:45-50.

14. Rudberg MA, Furner SE, Dunn JE et al. The relationship of visual and hearing impairments to disability: An analysis using the longitudinal study of aging. J Gerontol 1993;48:M261-M265.

15. Wallhagen MI, Strawbridge WJ, Kaplan GA. 6-year impact of hearing impairment on psychosocial and physiologic functioning [letter]. Nurse Pract 1996;21:11-14.

16. Kalina RE. Aging and visual function. In: Hazzard WR, Blass JP, Ettinger WH et al., eds. Principles of Geriatric Medicine and Gerontology. New York: McGraw-Hill, Inc., 1999, pp 603-616.

17. West SK, Munoz B, Rubin GS et al. Function and visual impairment in a population-based study of older adults. The SEE project. Salisbury Eye Evaluation. Invest Ophthalmol Vis Sci 1997;38:72-82.

18. Andersson G, Melin L, Scott B et al. An evaluation of a behavioural treatment approach to hearing impairment. Behav Res Ther 1995;33:283-292.

19. Loovis CF, Schall DG, Teter DL. The role of assistive devices in the rehabilitation of hearing impairment. Otolaryngol Clin North Am 1997;30: 803-847.

20. Brenner MH, Curbow B, Javitt JC et al. Vision change and quality of life in the elderly. Response to cataract surgery and treatment of other chronic ocular conditions. Arch Ophthalmol 1993; 111:680-685.

21. Demers-Turco P. Providing timely and ongoing vision rehabilitation services for the diabetic patient with irreversible vision loss from diabetic retinopathy. J Am Optom Assoc 1999;70:49-62.

22. Margrain TH. Helping blind and partially sighted people to read: The effectiveness of low vision aids. Br J Ophthalmol 2000;84:919-921.

23. Reuben DB, Mui S, Damesyn M et al. The prognostic value of sensory impairment in older persons [see comments]. J Am Geriatr Soc 1999;47:930-935.
24. Guralnik JM. The impact of vision and hearing impairments on health in old age [editorial comment]. J Am Geriatr Soc 1999;47:1029-1031.

25. Strawbridge WJ, Cohen RD, Shema SJ et al. Successful aging: Predictors and associated activities. Am J Epidemiol 1996;144:135-141.

26. Association AP. Diagnostic and Statistical Manual: Third Edition Revised (DSM-III-R). Washington, DC: American Psychiatric Association, 1987.

27. Walter SD, Feinstein AR, Wells CK. Coding ordinal independent variables in multiple regression analyses. Am J Epidemiol 1987;125:319-323.

28. Maclure M, Greenland S. Tests for trend and dose response: Misinterpretations and alternatives [see comments]. Am J Epidemiol 1992;135:96-104.

29. Eye care visits and use of eyeglasses or contact lenses. United States, 1979 and 1980. Vital Health Stat 1984;10(145):1-60.

30. Saaddine JB, Narayan V, Engelgau MM et al. Prevalence of self-rated visual impairment among adults with diabetes. Am J Public Health 1999;89:12001205.

31. Reuben DB, Walsh K, Moore AA et al. Hearing loss in community-dwelling older persons: National prevalence data and identification using simple questions. J Am Geriatr Soc 1998;46:1008-1011.

32. Carabellese C, Appollonio I, Rozzini R et al. Sensory impairment and quality of life in a community elderly population. J Am Geriatr Soc 1993;41:401-407.

33. Appollonio I, Carabellese C, Frattola L et al. Effects of sensory aids on the quality of life and mortality of elderly people: A multivariate analysis. Age Ageing 1996;25:89-96.

34. Dargent-Molina P, Hays M, Breart G. Sensory impairments and physical disability in aged women living at home. Int J Epidemiol 1996;25:621-629.

35. Reinhardt JP. The importance of friendship and family support in adaptation to chronic vision impairment. J Gerontol B Psychol Sci Soc Sci 1996;51:268-278.

36. Baltes PB, Lindenberger U. Emergence of a powerful connection between sensory and cognitive functions across the adult life span: A new window to the study of cognitive aging? Psychol Aging 1997;12:12-21.

37. Salthouse TA, Hancock HE, Meinz EJ et al. Interrelations of age, visual acuity, and cognitive functioning. J Gerontol B Psychol Sci Soc Sci 1996;51:317-330.

38. Luszcz MA, Bryan J. Toward understanding age-related memory loss in late adulthood. Gerontology 1999;45:2-9.

39. Schaie KW. Intellectual development in adulthood. In: Birren JE, Schaie KW, eds. Handbook of the Psychology of Aging. San Diego, CA: Academic Press, 1996, pp 266-286.

40. Gennis V, Garry PJ, Haaland KY et al. Hearing and cognition in the elderly. New findings and a review of the literature. Arch Intern Med 1991;151: 2259-2264.

41. Godlee F. Noise: Breaking the silence. BMJ 1992;304:110-113.

42. Chiodo AA, Alberti PW. Experimental, clinical and preventive aspects of ototoxicity. Eur Arch Otorhinolaryngol 1994;251:375-392.

43. Clark K, Sowers MR, Wallace RB et al. Age-related hearing loss and bone mass in a population of rural women aged 60 to 85 years. Ann Epidemiol 1995;5:8-14.

44. Gatland D, Tucker B, Chalstrey S et al. Hearing loss in chronic renal failurehearing threshold changes following haemodialysis. J Roy Soc Med 1991;84: 587-589.

45. Hariri MA, Lakshmi MV, Larner S et al. Auditory problems in elderly patients with stroke. Age Ageing 1994;23:312-316.

46. Lim DP, Stephens SD. Clinical investigation of hearing loss in the elderly. Clin Otolaryngol 1991;16:288-293.

47. Shusterman DJ, Sheedy JE. Occupational and environmental disorders of the special senses. Occup Med 1992;7:515-542.

48. Song BB, Schacht J. Variable efficacy of radical scavengers and iron chelators to attenuate gentamicin ototoxicity in guinea pig in vivo. Hear Res $1996 ; 94$ : 87-93.

49. Margrain TH. Minimising the impact of visual impairment. Low vision aids are a simple way of alleviating impairment [editorial]. Br Med J 1999;318:1504.

50. Rumney NJ. The aging eye and vision appliances. Ophthalmic Physiol Opt 1998;18:191-196. 